Supplement of Solid Earth, 10, 15-25, 2019

https://doi.org/10.5194/se-10-15-2019-supplement

(c) Author(s) 2019. This work is distributed under

the Creative Commons Attribution 4.0 License.

(c) (1)

Supplement of

\title{
The impact of earthquake cycle variability on neotectonic and paleoseismic slip rate estimates
}

\author{
Richard Styron \\ Correspondence to: Richard Styron (richard.h.styron@gmail.com)
}

The copyright of individual parts of the supplement might differ from the CC BY 4.0 License. 
This document contains the relevant results of two numerical experiments that are identical to those in the manuscript, except with somewhat different slip distributions. The first experiment uses an empirical slip distribution from Biasi and Weldon (2006) concatenated from 13 continental earthquakes and normalized to have a mean of 1 $\mathrm{m}$. This is perhaps a more realistic slip distribution than the lognormal distribution used in the experiment in the manuscript, but as an empirical distribution it is limited to values that have been observed. The second experiment uses an invariant slip of $1 \mathrm{~m}$ for each earthquake, to highlight the effects of the variation in earthquake timing on the results.

Here, the figures showing the slip rate estimates are included; the setup (order, axes, symbology, etc.) of these figures are identical to Figure 5 in the manuscript to allow for easy comparison of these results, which are the most important in the manuscript. Tables similar to Table 1 in the manuscript are also included, so that comparison of the 'numbers' can be done as well.

\section{Simulations with empirical offset distribution}

This experiment uses an empirical slip distribution (Figure S1) from Biasi and Weldon (2006). The distribution is finite, and is composed of $\sim 1400$ total values representing field measurements and values interpolated by those authors from between irregularly-spaced measurements from a set of 13 earthquakes, which have been normalized to the mean offset for each earthquake. The final distribution has a mean of 1 (taken as $1 \mathrm{~m}$ in this experiment) and a CV of 0.67 , very slightly below the $\mathrm{CV}$ of 0.75 for the lognormal slip distribution used in the experiment in the manuscript, which also has a mean of $1 \mathrm{~m}$.

The empirical distribution used here is qualitatively dissimilar to the lognormal distribution used in the manuscript. The mode of the distribution is zero, and the central part of the distribution (values between $\sim 0.1 \mathrm{~m}$ and $1.75 \mathrm{~m}$ ) all share a broadly similar relative probability (0.4-0.55). Values greater than $1.75 \mathrm{~m}$ have a much lower, and decreasing probability, until the maximum value of about $3 \mathrm{~m}$; the distribution has compact support and values above this are strictly impossible, unlike the lognormal slip distribution used in the manuscript which allows values to approach infinity, though at extremely low probabilities.

In this experiment, slip values are sampled randomly from the finite set of values in this distribution with replacement.

The results (Figure S2, Table S1) are nearly indistinguishable from those from the experiment in the manuscript. The sole observable difference is that the 5th percentile in the estimated slip rates for the clusted lognormal recurrence interval is above zero following about 20 mean earthquake cycles (20,000 years) in the experiment with the empirical slip distribution. All other metrics are near-identical. 


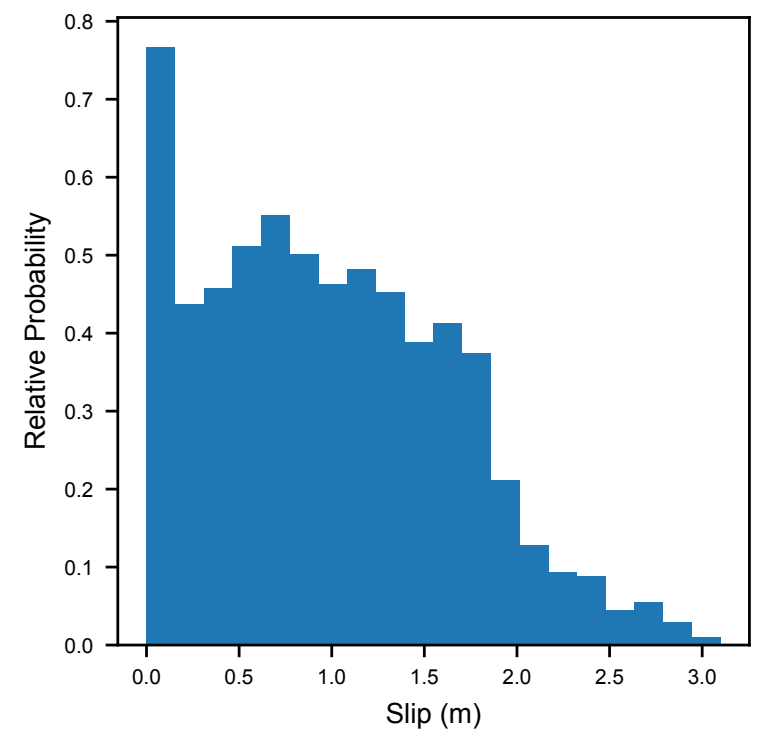

Figure S1: Histogram of per-event fault offsets from Biasi and Weldon (2006); compare this to Figure 3 in the manuscript.

\begin{tabular}{lllllll}
\hline distribution & $t$ & 5 & 25 & 50 & 75 & 95 \\
\hline lognormal, CV=0.5 & 2531 & 0.51 & 0.74 & 1.05 & 1.64 & 5.62 \\
& 4843 & 0.6 & 0.79 & 1.02 & 1.38 & 2.46 \\
& 10323 & 0.7 & 0.85 & 1 & 1.21 & 1.68 \\
& 42103 & 0.83 & 0.93 & 1 & 1.08 & 1.23 \\
lognormal, CV=1 & 2531 & 0.45 & 0.71 & 1.13 & 2.26 & $\infty$ \\
& 4843 & 0.53 & 0.77 & 1.09 & 1.65 & 4.94 \\
& 10323 & 0.64 & 0.84 & 1.06 & 1.39 & 2.32 \\
lognormal, CV=2 & 42103 & 0.8 & 0.93 & 1.04 & 1.17 & 1.48 \\
& 2531 & 0.35 & 0.59 & 1.16 & 8.44 & $\infty$ \\
& 4843 & 0.43 & 0.66 & 1.02 & 2.14 & $\infty$ \\
& 10323 & 0.52 & 0.72 & 0.96 & 1.55 & 6.04 \\
exponential, CV=1 & 42103 & 0.71 & 0.81 & 0.94 & 1.15 & 1.85 \\
& 2531 & 0.42 & 0.71 & 1.19 & 2.56 & $\infty$ \\
& 4843 & 0.51 & 0.77 & 1.1 & 1.77 & 7.02 \\
& 10323 & 0.61 & 0.84 & 1.07 & 1.41 & 2.35 \\
& 42103 & 0.81 & 0.95 & 1.04 & 1.15 & 1.39 \\
\hline
\end{tabular}

Table S1: Epistemic uncertainty table for Biasi and Weldon (2006) slip distribution

\section{Simulations with invariant $1 \mathrm{~m}$ offsets}

The results from the simulation without slip variability (Figure S3, Table S2) are broadly similar to the results from the simulation in the manuscript. The differences 

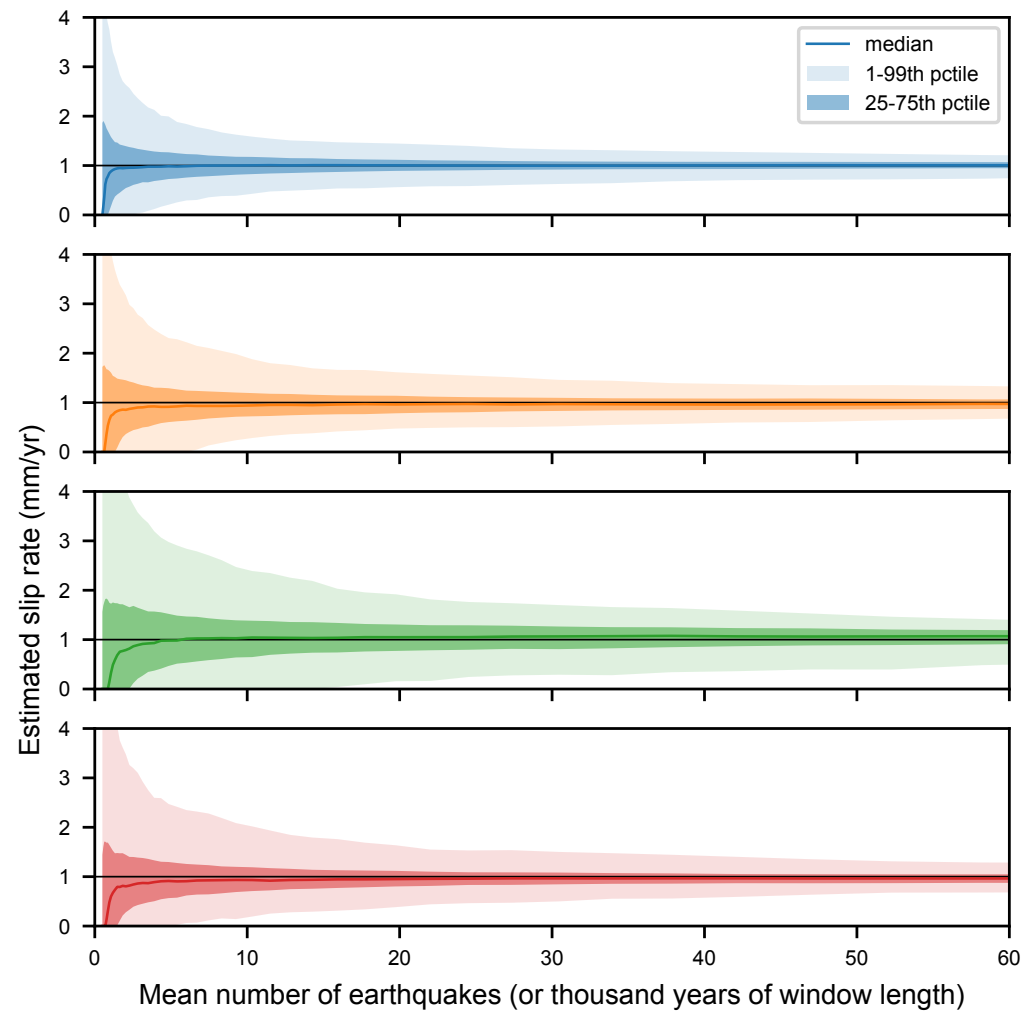

Figure S2: Variability in measured slip rates through time, using per-event fault displacements from Biasi and Weldon (2006) and all other parameters from the manuscript; compare this figure to Figure 5 in the manuscript. 
are that $a$ ) the effects of the position in the earthquake cycle on the measured slip rate cycle are much more clear for up to about 7 events, and $b$ ) that the variation in slip measurements at any time $t$, and the convergence of the slip estimates, is a bit different. These are both the expected results of removing a source of stochasticity or 'noise' from the system. With regards to $a$, the 'noise' from slip variability in the main experiment, which is uncorrelated with the variability in the earthquake recurrence time series, serves to smooth out the effects of the position within each early earthquake cycle. With regards to $b$, the variations in the slip rate estimates in this experiment at some time $t$ are a bit different than in the experiment in the manuscript: The total variability (as approximated by the 5th and 95th percentiles) is greater when slip variability is included (because there is more total stochasticity in the system) but these longer tails are thinner, and the central portion of the distributions (represented by the 25th, 50th, and 75th percentiles) are smoother and tighter, because of the smoothing effect of the slip variability.

With increasing time and cumulative earthquakes, the slip rate estimates in this experiment smooth out, and the variation is less at all percentiles than the experiment in the manuscript; this is consistent with less stochasticity in the system.

\begin{tabular}{lllllll}
\hline distribution & $t$ & 5 & 25 & 50 & 75 & 95 \\
\hline lognormal, CV=0.5 & 2531 & 0.63 & 0.84 & 0.84 & 1.27 & 2.53 \\
& 4843 & 0.69 & 0.81 & 0.97 & 1.21 & 1.61 \\
& 10323 & 0.79 & 0.94 & 1.03 & 1.15 & 1.29 \\
& 42103 & 0.9 & 0.96 & 1 & 1.05 & 1.14 \\
lognormal, CV=1 & 2531 & 0.51 & 0.63 & 0.84 & 1.27 & $\infty$ \\
& 4843 & 0.61 & 0.81 & 0.97 & 1.21 & 2.42 \\
& 10323 & 0.69 & 0.79 & 0.94 & 1.15 & 2.06 \\
lognormal, CV=2 & 42103 & 0.81 & 0.88 & 0.96 & 1.05 & 1.32 \\
& 2531 & 0.36 & 0.63 & 1.27 & $\infty$ & $\infty$ \\
& 4843 & 0.44 & 0.69 & 0.97 & 2.42 & $\infty$ \\
& 10323 & 0.52 & 0.74 & 1.03 & 1.72 & 10.32 \\
exponential, CV=1 & 42103 & 0.66 & 0.83 & 1 & 1.28 & 2.11 \\
& 2531 & 0.51 & 0.63 & 1.27 & 2.53 & $\infty$ \\
& 4843 & 0.54 & 0.81 & 0.97 & 1.61 & 2.42 \\
& 10323 & 0.65 & 0.79 & 1.03 & 1.15 & 1.72 \\
& 42103 & 0.77 & 0.9 & 0.98 & 1.08 & 1.24 \\
\hline
\end{tabular}

Table S2: Epistemic uncertainty table for invariant $1 \mathrm{~m}$ slip distribution

However, it is important to note that the differences between the results from this experiment and the experiment in the manuscript are minor. Perhaps most importantly, the relative differences between the recurrence distributions are maintained here, and the number of earthquakes required for the measured slip rates to stabilize are about the same. 

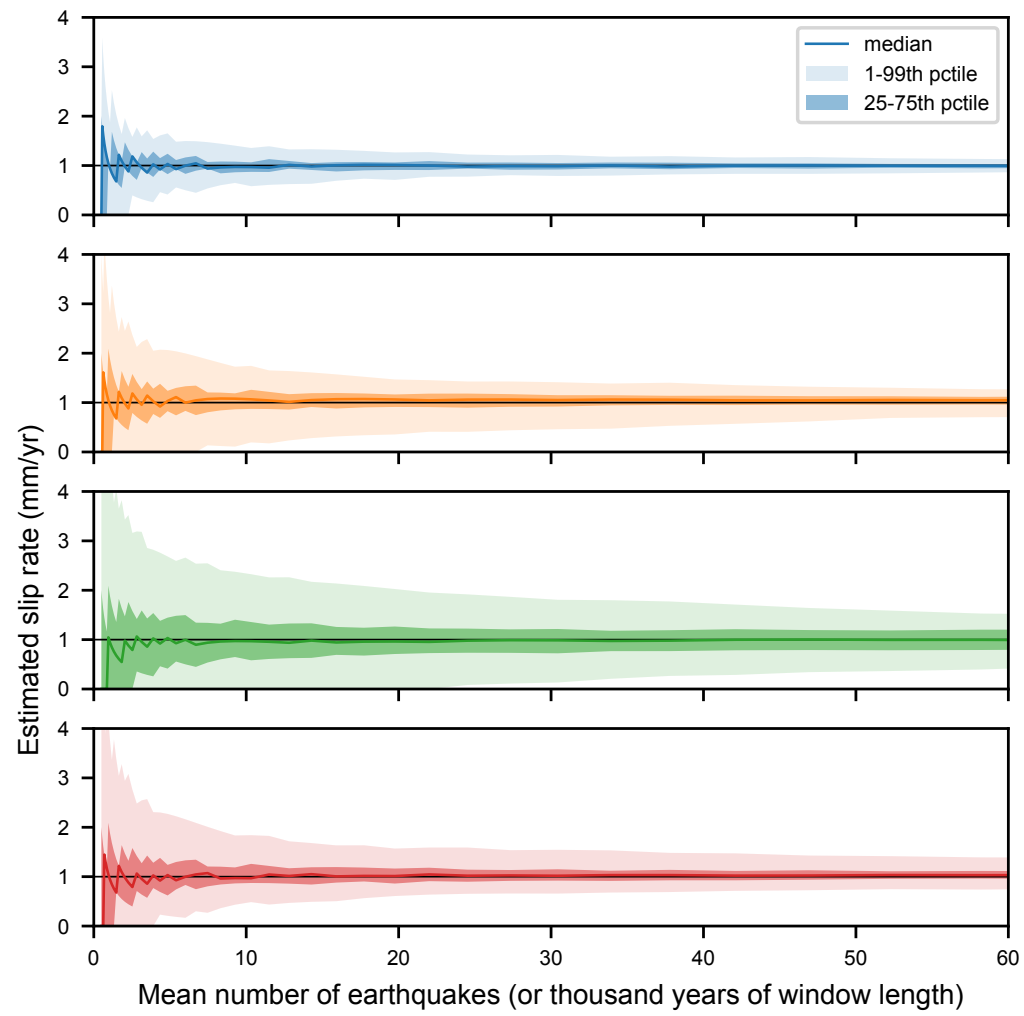

Figure S3: Variability in measured slip rate through time with invariant per-event offsets of $1 \mathrm{~m}$, and all other parameters from the manuscript; compare this to Figure 5 in the manuscript. 